\title{
Die Bestimmung von Sulfat als Baryumsulfat. Von
}

\section{J. M. Kolthoff und E. H. Vogelenzang.}

Wie noch neulich von $\mathrm{Karaoglanow}{ }^{1}$ ) betont worden ist, ist sine Sulfatbestimmung als Baryumsulfat mit vielen Mängeln behaftet. Zumal wirkt die Gegenwart von Elektrolyten störend, weil die Realstion dańn nicht völlig nach der Gleichung:

$$
\mathrm{Ba}{ }^{*}+\mathrm{SO}_{4}{ }^{\prime \prime} \rightarrow \mathrm{BaSO}_{4}
$$

vor sich geht. Die Elektrolyten bilden mit den $\mathrm{Ba}$ "- oder $\mathrm{SO}_{4}{ }^{\prime \prime}$-Ionen oder mit beiden in grösserem oder geringerem Malse komplexe Ionen, welche mit einander oder mit den Elementarionen in Verbindung treten and auf diese Weise den Niederschlag verunreinigen.

Zudem können andere Fehler auftreten, welche die Resultate ungünstig beeinflussen. In der folgenden Abhandlung sollen die verschiedenen Fehlerquellen besprochen werden.

Löslichkeit des Baryumsulfats.

Die Löslichkeit des Baryumsulfats in Wasser wird beherrscht durch das Löslichkeitsprodulkt:

$$
\left[\mathrm{Ba}^{*}\right] \cdot\left[\mathrm{SO}_{4}{ }^{\prime \prime}\right]=0,9 \times 10^{-10}\left(18^{\circ}\right) \text {. }
$$

Die zweite Dissoziationskonstante der Schwefelsäure ist ziemlich klein, nämlich $1,7 \times 10^{-2}$. Wenn man also zu einer Lösung, welche $\mathrm{SO}_{4}$-Ionen enthält, Wasserstoffionen hinzufügt, vereinigen beide sich nach der folgenden Reaktion:

$$
\mathrm{SO}_{4}^{\prime \prime}+\mathrm{H}^{\circ} \leftrightarrows \mathrm{HSO}_{4}^{\prime} \text {. }
$$

Weil also auf diese Weise Sulfationen entfernt werden, wird die Löslichkeit des Baryumsulfats in Säuren grösser als in Wasser sein. Aber auch durch Salze wird die Löslichkeit erhöht. Entweder können die Baryumionen mit den Anionen des zugesetzten Salzes:

$$
\begin{aligned}
& \mathrm{Ba}^{*}+\mathrm{Cl}^{\prime} \leftrightarrows \mathrm{Ba} \mathrm{Cl}^{\circ} \\
& \mathrm{Ba}{ }^{*}+\mathrm{NO}_{3}{ }^{\prime} \leftrightarrows \mathrm{BaNO}_{3} .
\end{aligned}
$$

oder die Sulfationen mit den Kationen des zugesetzten Salzes reagieren:

$$
\mathrm{SO}_{4}^{\prime \prime}+\mathrm{K}^{\cdot} \leftrightarrows \mathrm{K} \mathrm{SO}_{4}^{\prime} \text {. }
$$

1) Diese Ztschrft. 56, 417 (1917).

Fresenius Zeitschrift f. anal. Chemie. LVIII. 2. Heft. 4 
Bei steigender Temperatur wird die Löslichkeit grösser. Durcis Hinzufügung von gleichnamigen Ionen wird der Theorie nach die Löslichkeit des Baryumsulfats stark herabgesetzt.

Weil die verschiedenen Gleichgewichtskonstanten der obengenannten Reaktionen nicht bekannt sind, kann man die verschiedenen Löslichkeitserhöhungen durch Säuren und Salze nicht voraus berechnen. Wir werden darum im folgenden nur die praktischen Resultate mitteilen.

Die Löslichkeit des Baryumsulfats in Wasser ist von verschiedenen Autoren bestimmt worden. In folgender Tabelle sind die verschiedenen Resultate angegeben. Zudem ist die Methode zur Bestimmung der Löslichkeit mitgeteilt.

\begin{tabular}{|c|c|c|c|}
\hline Autor & Methode & $m g$ in $1 . l$ & Temperatur \\
\hline Eresenius ${ }^{1}$ ) . . . . & direkt & 2,5 & - \\
\hline Fresenius und Hintz & indirekt & 10 & - \\
\hline$\left.K u ̈ s t \in r^{3}\right) \ldots . .$. & Leitfähigkeit & 2,35 & $18,3^{\circ}$ \\
\hline Hollemann $n^{4}$. . . . & $n$ & 2,33 & $18,4^{0}$ \\
\hline n $\quad . \quad .$. & * & 3,12 & $37,7^{\circ}$ \\
\hline Kohlrausch u. Roses). & 》 & 1,7 & 2,00 \\
\hline$r$ & » & 2,0 & 10.00 \\
\hline$\pi$ & $n$ & 2,3 & $19,0^{\circ}$ \\
\hline , & , & 2,6 & $26,0^{0}$ \\
\hline * & " & 2,9 & $34,0^{0}$ \\
\hline$n$ & , & 3,1 & $37,7^{\circ}$ \\
\hline
\end{tabular}

Von diesen Methoden ist die durch Leitfähigkeitsbestimnung die genaueste.

Es ist jedoch zu bemerken, dass die Löslichkeit des Baryumsulfats keine konstante Grösse ist, dass sie vielmehr von der Korngrösse des Präparates abhängig ist.

So fand $\mathrm{Hulett}^{6}$ ), dass die Bestimmungen von $\mathrm{K}$ üster, Elollemann und Kohlrausch-Rose sich bezogen auf Präparate, deren Korngrösse mehr als $1,8 \mu$ war. Bei sehr kleiner Korngrösse $(0,1 \mu)$ fand er eine Löslichkeit von $4,15 \mathrm{mg}$ in 17 .

1) Diese Ztschrft. 9, 52 (1870).

2) Ebenda 35, 170 (1896).

3) Ztschrft. f. anorg. Chem. 12, 261 (1896).

4) Ztschrft. f. physik. Chem. 12, 125 (1893); diese Ztschrft. 42, 756 (1903).

5) Ebenda 12, 131, 241 (1893); diese Ztschrft. 42, 756 (1903).

6) Ztschr. f. physik. Chem. 37, 385 (1901). 
Wir haben auch die Löslichkeit von reinem Baryumsulfat von verschiedener Korngrösse bestimmt. Die Präparate wurden aus reinem Baryt und Schwefelsäure unter solchen Bedingungen dargestellt, wie sie auch bei der Analyse vorkommen können. Auf Reinigung der Präparate und weitere Einzelheiten bei den Löslichkeitsbestimmungen wird hier nicht weiter eingegangen werden.

$A$ wurde dargestellt indem man zu einer gesättigten Barytlösung bei Siedehitze so lange $4 \mathrm{n}$-Schwefelsäure hinzufügte, bis die Reaktion auf Methylrot sauer war. Das weiter gereinigte Präparat wurde bei $40^{\circ}$ getrocknet.

$B$ wurde aus A hergestellt indem man A längere. Zeit mit verdünnter Salzsäure kochte (Umkristallisation). Das Präparat wurde ausgewaschen und getrocknet.

C Ein Teil von B wurde kurze Zeit in einem Porzellantiegel geglüht.

$D$ wurde analog wie $A$ erhalten, jedoch wurde bei Zimmertemperatur gefällt.

E Ein Teil von D wurde kurze Zeit geglüht.

Die Präparate wurden mit Leitfähigkeitswasser bei $18^{\circ} \mathrm{im}$ Thermostaten geschüttelt. Nach dem Absetzen wurde dann die Leitfähigkeit bei $18^{\circ}$ bestimmt. Die Leitfähigkeit des angewandten Wassers wurde in Abzug gebracht. Nach dem Abgiessen des Wassers wurden die Präparate wieder mit frischem Wasser geschüttelt and dies so lange wiederholt, bis die Leitfähigkeit konstant blieb.

\begin{tabular}{c|c|c}
\hline \hline Präparat & $K_{18} \times 10^{6}$ & $\begin{array}{c}\text { Löslichkeit } \\
m g \text { in } 1 l\end{array}$ \\
\hline & 2,25 & 2,11 \\
B & 2,50 & 2,35 \\
C & 2,20 & 2,07 \\
D & 2,40 & 2,25 \\
E & 2,69 & 2,53
\end{tabular}

Nach der Erwartung ist die Löslichkeit von A geringer als von D.

Löslichkeit in verdünnten Säuren.

a) Salzsäure. Die Löslichkeit von Baryumsulfat in Salzsäure verschiedener Konzentration ist von Ostwald-Banthisch ${ }^{1}$ ) und Allen-Johnston ${ }^{2}$ ) bestimmt worden. Es ist zu bedauern, dass

1) Journ. f. prakt. Chem. 1884, S. 53; diese Ztschrft. 56, 241 (1917).

2) Journ. Americ. Chem. Soc. 32, 588; diese Ztschrft. 50, 182 (1911). 
Allen und Johnstion die Versuchstemperatur nicht angeben, weil die Löslichkeit des Baryumsulfats mit der Temperatur stark schwankt. Doch sind die Differenzen zwischen den Resultaten der genannten Autoren sebr gross, wie folgende Tabelle zeigt:

\begin{tabular}{|c|c|c|c|}
\hline \multirow[b]{2}{*}{$\mathrm{N}$} & \multicolumn{2}{|c|}{$\begin{array}{l}\text { Löslichkeit des } \mathrm{BaSO}_{4} \\
m g \text { in } 1 l\end{array}$} & \multirow[b]{2}{*}{ Temperatur } \\
\hline & $\mid \begin{array}{c}\text { nach } \\
\text { Ostwald- } \\
\text { Banthisch }\end{array}$ & $\begin{array}{c}\text { mach } \\
\text { Allen - } \\
\text { Johnston }\end{array}$ & \\
\hline 0,003 & - & 2,6 & - \\
\hline 0,016 & - & 4,3 & - \\
\hline 0,08 & - & 9,4 & - \\
\hline 0,16 & - & 11,1 & - \\
\hline 0,3 & - & 34,3 & - \\
\hline 0,5 & 67 & - & $20^{\circ}$ \\
\hline 1,0 & 89 & - & $20^{\circ}$ \\
\hline 1,5 & - & 103 & - \\
\hline 2,0 & 101 & - & 200 \\
\hline 5,0 & 86 & - & $20^{\circ}$ \\
\hline
\end{tabular}

Wir haben auch einige Bestimmungen ausgeführt. Weil sich die Resultate stark von den in der Tabelle verzeichneten unterscheiden, werden sie unten wiedergegeben werden.

Aus reiner Salzsäure wurden verschiedene Verdünnungen genau hergestellt and nacher der Titer derselben bestimmt. $0.5 \mathrm{~g}$ Baryumsulfat (Präparat 'D) wurden mit $250 \mathrm{ccm}$ der Säure in Köln-EhrenfeldFlaschen bei $12^{\circ} 7-8$. Stunden geschüttelt. Nach dem Absetzen wurde unter den nötigen Vorsichtsmaßregeln filtriert. Ein Teil des Filtrats wurde in einer Platinschale eingedampft, geglüht und gewogen. In dieselbe Schale wurde dann wieder ein Teil des Filtrats gebracht und das Eindampfen etc. wiederholt.

Die Resultate sind folgende:

\begin{tabular}{|c|c|c|c|c|}
\hline & $\begin{array}{c}\text { Filtrat } \\
\mathrm{cem}\end{array}$ & $\begin{array}{c}\text { Rückstand } \\
m g\end{array}$ & $\begin{array}{l}\text { Löslichkeit } \\
m q \text { in } 1 l\end{array}$ & $\begin{array}{c}\text { Mittel } \\
m g \text { in } 1 l\end{array}$ \\
\hline 0,98 normal & $\begin{array}{l}50 \\
45\end{array}$ & $\begin{array}{l}2,7 \\
2,4\end{array}$ & $\begin{array}{l}54 \\
53,6\end{array}$ & 53,8 \\
\hline 0,49 & $\begin{array}{l}50 \\
50\end{array}$ & $\begin{array}{l}1,7 \\
1,4\end{array}$ & $\begin{array}{l}34 \\
28\end{array}$ & 31 \\
\hline 0,098 & $\begin{array}{r}100 \\
50\end{array}$ & $\begin{array}{l}1,0 \\
0,6\end{array}$ & $\begin{array}{l}10 \\
12\end{array}$ & 11 \\
\hline
\end{tabular}


Unsere Resultate sind also merklich niedriger als die der genannten Autoren, ausgenommen in $\mathrm{n} / 10^{-\mathrm{HCl}}$ : Bemerkt, werden soll, dass unsere Bestimmungen bei viel niedrigerer Temperatur $\left(12^{\circ}\right)$ ausgeführt worden sind als die von Ostwald-Banthisch $\left(20^{\circ}\right)$.

Auch wurde die Löslichkeit bei $90^{\circ}$ bestimmt.

Das Baryumsulfat wurde mit Wasser oder Säure in einem Jenaer Kolben bis zum Sieden erbitzt, dann wurde der Kolben noch eine Stunde in ein siedendes Wasserbad gestellt (Thermometer im Kolben).

Darauf wurde filtriert and weiter wie oben verfahren.

\begin{tabular}{|c|c|c|c|}
\hline & $\begin{array}{c}\text { Filtrat } \\
\text { ccm }\end{array}$ & $\begin{array}{c}\text { Rückstand } \\
m g\end{array}$ & $\begin{array}{l}\text { Löslichkeit } \\
m g \text { in } 1 l\end{array}$ \\
\hline Wasser . & $\begin{array}{c}50 \\
\text { aus Leitf. }\end{array}$ & $\begin{array}{c}0,35 \\
=6 \times 10-6\end{array}$ & $\begin{array}{l}7 \\
5,6\end{array}$ \\
\hline 1,00 normal & $\begin{array}{l}50 \\
50\end{array}$ & $\left.\begin{array}{l}18,9 \\
22,4\end{array}\right\}$ & 41,6 \\
\hline $0, \breve{500}$ & $\begin{array}{l}50 \\
50\end{array}$ & $\left.\begin{array}{l}13,8 \\
12,7\end{array}\right\}$ & 26,5 \\
\hline 0,05 & $\begin{array}{l}50 \\
50\end{array}$ & $\left.\begin{array}{l}3,7 \\
4,0\end{array}\right\}$ & 7,7 \\
\hline
\end{tabular}

In heisser Salzsäure ist die Löslichkeit also sehr gross. Auch wurde dem Einfluss zugefügter Baryumionen nachgegangen. $\mathrm{Zu}$ der Säurelösung, wurden $0,5 \% \quad \mathrm{BaCl}_{2} \cdot 2 \mathrm{H}_{2} \mathrm{O}$ hinzugefügt; dann wurde mit Baryumsulfat 7 Stunden lang geschüttelt. Nach dem Absetzen wurde filtriert und ein Teil des Filtrats eingedampft. Der Rückstand warde mit Wasser aufgenommen, auf einem kleinen Filter gesammelt, ausgewaschen und noch nass in einem Mikro-Neubauer-Tiegel geglüht.

Dann wurde ein Tropfen verdünnter Schwefelsäure hinzugefügt (Regeneration des Sulfids zu Sulfat), ausgewaschen und von neuem schwach geglüht. 'Schliesslich" wurde auf einer Mikrowage von Kuhlmann gewogen.

\begin{tabular}{|c|c|c|c|c|c|}
\hline N. & & $\frac{\mathrm{BaCl}_{2}}{\text { konzentriert }}$ & $\begin{array}{c}\text { Filtrat } \\
\text { ecm }\end{array}$ & $\begin{array}{c}\text { Rückstand } \\
m g\end{array}$ & $\begin{array}{c}\text { Löslichk. bei } 20^{\circ} \\
m a \text { in } 1 l\end{array}$ \\
\hline $\begin{array}{l}\text { Normal } \\
0,5 \text {-normal }\end{array}$ & $\begin{array}{l}\cdot \quad \cdot \quad \cdot \\
\cdot \quad \cdot \quad \cdot\end{array}$ & $\begin{array}{l}0,04 n \\
0,04 \mathrm{n}\end{array}$ & $\begin{array}{r}50 \\
100\end{array}$ & $\begin{array}{l}1,79 \\
1,34\end{array}$ & $\begin{array}{l}35,8 \\
13,4\end{array}$ \\
\hline
\end{tabular}

Die Löslichkeit wird also stark herabgesetzt. 
b) Löslichkeit in Salpetersäure. Die Löslichkeit in Salpetersăure wurde genau wie die in Salzsäure bestimmt. In folgender Tabelle sind die Resultate bei $19^{\circ}$ angegeben. Zum Vergleich sind in der Reihe 0 . B. die von 0 stwald und $B$ anthisch erhaltenen Werte angeführt.

\begin{tabular}{|c|c|c|c|c|c|c|c|}
\hline & & . & & $\begin{array}{c}\text { Filtrat } \\
\mathrm{ccm}\end{array}$ & $\begin{array}{c}\text { Rückstand } \\
m g \\
\end{array}$ & $\begin{array}{c}\text { Löslichk. bei } 190 \\
m g \text { in } 1 l\end{array}$ & $\begin{array}{c}0 . \text { B. bei } 20^{\circ} \\
m g \text { in } 17\end{array}$ \\
\hline 2 fach norma & & $\because$ & . & $\begin{array}{l}50 \\
50\end{array}$ & $\left.\begin{array}{l}6,2 \\
5,7\end{array}\right\}$ & 11,9 & 170 \\
\hline normal & . & . & . & $\begin{array}{l}50 \\
45\end{array}$ & $\left.\begin{array}{l}4,3 \\
3,0\end{array}\right\}$ & 7,7 & 107 \\
\hline 0,5-normal & . & . & . & $\begin{array}{l}50 \\
50\end{array}$ & $\left.\begin{array}{l}1,8 \\
2,1\end{array}\right\}$ & 3,9 & 70 \\
\hline $\begin{array}{l}0,1 \\
0,05\end{array}$ & . & $\begin{array}{l}\cdot \\
\cdot\end{array}$ & $\dot{.}$ & $\begin{array}{l}100 \\
100\end{array}$ & $\begin{array}{c}1,2 \\
(0,9)\end{array}$ & $\begin{array}{l}1,2 \\
(9)\end{array}$ & - \\
\hline
\end{tabular}

Auch hier finden wir eine geringere Löslichkeit als 0 stwald und Banthisch.

c) Reduktion des Niederschlags. Während der Untersuchung zeigte es sich, dass bei trockner Veraschung des Filters im Platintiegel starke Reduktion eintritt, in Porzellantiegeln dagegen in viel geringerem Maße. Wenn man das Filter noch feucht verascht, so findet fast gar keine Reduktion statt. Aus einem dazu angestellten Versuch ergab sich, dass beim Glühen einer Mischung von Baryumsulfat mit $1 / 20$ seines Gewichtes an Kohle ein Gewichtsverlust ron $0,6 \%$ eintrat. Glühen an der Luft war nicht genügend, diesen Fehler zu beseitigen.

Wenn man jedoch nach dem Glühen einige Tropfen Schwefelsäure hinzusetzt und wieder erhitzt, wird der Reduktionsfehler beseitigt.

Zudem ergab sich, dass zur Abkühlung eines Porzellantiegels ein Exsikkator unnötig ${ }^{1}$ ), ja sogar zu verwerfen ist.

Wenn ein Tiegel von $24 \mathrm{~g} 10$ Minuten geglüht wurde, war das Gewicht nach $1 \frac{1}{2}$ stündigem Stehen an der Luft konstant.

1) Winkler, Ztschrft. f. angew. Chem. 30, 251, 259 (1917); diese Ztschrft. อ̃7, 234 (1918). 
Über die Okklusion ron Salzen.

Unter okkludierten Stoffen verstehen wir alle fremden Stoffe, die vom Baryunsulfat mitgerissen werden.

Dieses Einschliessen oder Okkludieren fremder Stoffe kann anf verschiedene Weise geschehen.

a) Unter bestimmten Umständen kann der okkludierte Stoff mit dem Baryumsulfat ein Konglomerat bilden. Dies ist u. a. mit Baryumphosphat der Fall, wie sich später zeigen wird.

b) Der Stoff befindet sich in einer festen Lösung.

c) Der Stoff wird vom Baryumsulfat adsorbiert.

b und c sind praktisch ungefähr dasselbe. Wenn man nämlich Baryumsulfat mit Elektrolyten schüttelt, dann müsste ein Teil der letzteren verschwinden, wenn eine Wirkung nach b oder $c$ stattfände. Aus Versuchen; die wir mit yerschieden konzentrierten Salzlösungen ( $\mathrm{Fe} \mathrm{Cl}_{3}, \mathrm{HCl}$ usw.) anstellten, zeigte es sich, dass das Baryumsulfat der Lösung keinen Elektrolyt entzog. Nur kamen bei Eisenchlorid in neutraler Lösung Abweichungen vor, die aber nicht durch Adsorption verursacht wurden.

Dass Baryumsulfat. keine kristallinischen Stoffe adsorbiert, entspricht auch der Untersuchung von Robert Marc, der zu dem Schluss kam, dass kristallinische Stoffe wohl Kolloide adsorbieren, aber nur diejenigen Kristalloide, die mit dem Adsorbens isomorph sind. Wir bekommen dann den Fall

d) Der okkludierte Stoff bildet mit dem Baryumsulfat Mischkristalle. Es ist aber nicht wahrscheinlich, dass die Okklusion im allgemeinen der Bildung von Mischkristallen zuzuschreiben ist, da die am meisten vorkommenden störenden Salze mit Baryumsulfat nicht isomorph sind und sogar zu einem andern Kristallsystem gehören.

$\mathrm{BaSO}_{4}$ ist rhombisch

$\mathrm{BaCl}_{2} \gg$ monoklinisch

$\mathrm{Ca} \mathrm{Cl}_{2}-6$ aq ist trigonal

$\mathrm{Ba}\left(\mathrm{NO}_{3}\right)_{2}$ ist regulär
$\mathrm{Fe} \mathrm{Cl}_{3}$ ist hexagonal (trigonal)

$\mathrm{NaCl}$ \& regulär

$\mathrm{K} \mathrm{Cl} \approx$ regulär

$\mathrm{Na} \mathrm{NO}_{3}$ ist ditrigonal.

e) Der Stoff wird mechanisch eingeschlossen.

Aus Versuchen, die wir später angestellt haben, und wobei der Feinheitsgrad des Niederschlags geändert warde, zeigte es sich, 
dass, wenn auch ein Einschliessen stattfindet, diesem nar zum Teil der Fehler 'der Okklusion zuzuschreiben sein kann.

f) Die Okklusion findet statt infolge einer chemischen Reaktion. Wie wir sehen werden, ist diese Annahme die wahrscheinlichste. Die ternären Elektrolyte wie Baryumchlorid oder Kaliumsulfat, werden nicht auf einmal, sondern stufenweise in einfache Ionen. gespalten:

$$
\text { z. B. } \begin{aligned}
& \mathrm{BaCl}_{2} \underset{\mathrm{BaCl}}{\rightleftarrows}+\mathrm{Cl}^{\prime} \\
& \mathrm{BaCl}^{\circ} \rightleftarrows \mathrm{Ba}^{*}+\mathrm{Cl}^{\prime} \text {. }
\end{aligned}
$$

Wenn viele $\mathrm{BaCl}$-ionen anwesend sind, so vereinigen diese sich mit $\mathrm{SO}_{4}{ }^{\prime \prime}$ :

$$
2 \mathrm{BaCl}^{\circ}+\mathrm{SO}_{4}{ }^{\prime \prime} \rightarrow \mathrm{Ba}_{2} \mathrm{Cl}_{2} \mathrm{SO}_{4},
$$

wodurch zuviel gefunden wird.

Durch die teilweise Spaltung des Kaliumsulfats in $K^{\prime}$ und $\mathrm{KSO}_{4}^{\prime}$ kann die Reaktion stattfinden:

$$
\text { 2. } \mathrm{KSO}_{4}^{\prime}+\mathrm{Ba} \rightarrow \mathrm{BaK}_{2}\left(\mathrm{SO}_{4}\right)_{2} \text {, }
$$

wodurch zu wenig gefunden wird.

In diesem Abschnitt werden die Störungen, welche durch Baryumchlorid, Nitrate, Phosphate, Kalzium-, Ferri- und einige andere Metall-Ionen verursacht werden; besprochen werden.

a) Baryumchlorid. Dieser Fehler ist von Richards und Parke $\mathbf{r}^{1}$ ) beobachtet worden. Sie fanden, dass die Okklusion von Baryumchlorid eine grosse Abweichung verursacht, welche um so grösser wird, je konzentrierter die Lösung und je mehr Salzsäure anwesend ist: Diese Erscheinungen entsprechen der Annahme, dass Baryumehlorid stufenweise dissozilert ist. (Siehe unter Okklusion.)

Bei grösserer Konzentration an Baryumchlorid nimmt die $\mathrm{BaCl}$ lonenkonzentration verhältnismälsig mehr zu als die Ba "-Ionenkonzentration, so dass mehr $\mathrm{BaCl}$-Ionen mit $\mathrm{SO}_{4}{ }^{\prime \prime}$-Ionen reagieren können. Dieselbe. Wirkung wird durch : Hinzufügen von viel $\mathrm{Cl}^{\prime}$-Ionen, d. h. hier Salzsäure, erreicht, wodurch mehr BaCl-Ionen entstehen.

In Übereinstimmung mit obiger Erklärung ist die Bemerkung von Karaoglanow ${ }^{2}$ ), dass der Fehler abnimmt bei grösserer Verdünnung; in diesem Falle sind aber mehr einfache Ionen anwesend. Aus demselben Grunde soll man immer. möglichst warm fällen.

1) Diese Ztschrft. 33, 43 (1894).

2) Diese Ztsehrft. 56, 417 (1917). 
Allen und Johuston ${ }^{1}$ ) erklären jedoch, dass die Okklusion von Baryumchlorid keine Fehler verursachen kann, da nach ihnen das Chlor beim Erhitzen des Niederschlags entweicht.

Wir konnten in all unseren Niederschlägen durch Schmelzen mit Soda $\mathrm{Cl}^{*}$ nachweisen und können also die Beobachtung von Allen und $J \circ h n s t o n$ nicht bestätigen.

Schliesslich bemerkt Pregl ${ }^{2}$ ), dass das okkludierte Baryumchlorid nach dem Glühen leicht ausgewaschen werden kann.

Diese Eigenschaft desselben würde hinweisen auf Oklklusion der Mutterlauge in Kristallhohlräumen, welche: durch das Erhitzen geöffnet worden sind. Es leuchtet ein, dass der Grad dieser Okklusion von der Korngrösse des Niederschlags abhängt und nur bei sehr grob kristallinischem Baryumsulfat bedeutend werden kann:

Dass bei einem feinkörnigen Niederschlag diese Okklusion nicht eintritt, wurde in folgender Weise nachgewiesen: In einem Platintiegel wurde bei Siedehitze eine Natriumsulfatlösung mit einem Überschuss der Baryumchloridlösung gefällt. 10-15 Minuten nach der Fällung filtrierte man durch einen Mikro-Neubauer-Tiegel, in welchem der Niederschlag ausgewaschen wurde. Nach dem Trocknen wurde schwach geglüht und nach Abkühien auf einem Aluminiumblock in einen Exsikkator wurde der Tjegel auf einer Mikrowage von Kuhlmann. gewogen. Hiernach wurde nochmals ausgewaschen, getrocknet, geglüht und gewogen.

Die Ergebnisse sind unten mitgeteilt.

Nr. 1, war in nentraler Lösung gefällt, aber auf dem Filter mit wenig verdünnter Salzsäure gewaschen.

Nr. 2 ebenfalls neutral niedergeschlagen, aber sofort nach der Fällung wurde ein Tropfen $25 \%$ iger Salzsäure hinzugefügt und weiter wie oben verfahren.

Nr. 3 wurde in schwach saurer Lösung gefält und weiter wie 1 behandelt.

$A$ ist das Gewiclit nach dem Glühen, B das nach dem Auswaschen und Glühen.

1) A. a. $O$.

2) Die quantitative organische Mikroanalyse (1917), S. 29 . 


\begin{tabular}{|c|c|c|c|c|}
\hline \multirow{3}{*}{ Nr. } & \multicolumn{3}{|c|}{$\mathrm{BaSO}_{4}$ gefunden } & \multirow{3}{*}{$\begin{array}{c}\mathrm{BaSO}_{4} \\
\text { berechnet } \\
m g\end{array}$} \\
\hline & A & $\cdot \mathrm{B}$ & $A-B$ & \\
\hline & $m g$ & $m g$ & $m g$ & \\
\hline 1 & 5,07 & 5,05 & 0,02 & 4,30 \\
\hline 2 & 4,63 & 4,60 & 0,03 & 4,30 \\
\hline 3 & 5,06 & 5,01 & 0,05 & 4,30 \\
\hline
\end{tabular}

In unserem Falle ist die Differenz $A-B$ nur von geringer Bedeutung, da sie den Wägefehler kaum überschreitet.

Von unkristallisiertem reinem Natriumsulfat, das 6 Monate bei einer relativen Dampfspannung von $85 \%$ aufbewahrt war und die Zusammensetzung $\mathrm{Na}_{2} \mathrm{SO}_{4} \cdot 10 \mathrm{H}_{2} \mathrm{O}$ hatte, wurde eine Lösung hergestellt indem man eine genau gewogene Menge in einen Maßkolben brachte und mit destilliertem Wasser anfüllte. Die Stärke der Lösung wurde jedesmal so gewählt, dass $25 \mathrm{ccm} 0,4 \mathrm{~g} \mathrm{Ba} \mathrm{SO} \mathrm{B}_{4}$ entsprachen.

Für jeden Versuch wurde diese Menge immer mit derselben Pipette abgemessen.

Dann fügte man Wasser und die verschiedenen Reagenzien hinzu, erhitzte zum Sieden und liess unter gelindem Umrühren aus einer Pipette eine $10 \%$ ige $\mathrm{BaCl}_{2}-2 \mathrm{H}_{2} \mathrm{O}$-Lösung hinzutröpfeln, während die Flüssigkeit immer schwach kochte. Den erhaltenen Niederschlag liess man auf einem Wasserbad absitzen und oft über Nacht steben. Danach wurde derselbe auf einem Filter (Schleicher und Schüll 589) gesammelt, nachdem er erst durch dreimaliges Dekantieren gewaschen war. Auf dem Filter wurde er weiter auf Chlorreaktion ausgewaschen, getrocknet und in einem Porzellantiegel verascht. Später wurde er auch wohl trocken oder feucht in einem Platintiegel verbrannt. In letzterem Falle (Veraschen des trockenen Niederschlags im Platintiegel) fand starke Reduktion des Baryumsulfats statt (starker Schwefelwasserstoffgeruch nach dem Ansäuern). Zur Regeneration rauchte man nach dem Glühen mit einigen Tropfen Schwefelsäure $a b$ and glübte danu wieder bis zu konstantem Gewicht.

Karaoglanow ${ }^{1}$ ) fand dagegen koine Reduktion, 'wenn er das Filter in einem Platintiegel verbrannte, wohl aber beim Veraschen im Porzellantiegel. Bei Erwähnung der Ergebnisse der Analyse werden

1) Diese Ztschrft. ธ6, 225. (1917). 
wir die Umstände, die gefundene und berechnete Menge Baryumsulfat und den relativen Fehler $\frac{\mathrm{O}-\mathrm{C}}{\mathrm{C}}$ in Prozenten angeben, wenn 0 die gefundene Menge, $\mathrm{C}$ die berechnete Menge $\mathrm{BaSO}_{4}$ ist.

\begin{tabular}{|c|c|c|c|c|c|c|c|c|}
\hline \multirow{3}{*}{$\mathrm{Nr}}$. & \multirow{3}{*}{$\begin{array}{l}\mathrm{SO}_{4}{ }^{\prime \prime}- \\
\text { Lösung } \\
\mathrm{ccm}\end{array}$} & \multirow{3}{*}{$\begin{array}{c}\text { Wasser } \\
\text { ccm }\end{array}$} & \multirow{3}{*}{$\begin{array}{c}4 \mathrm{n} \cdot \mathrm{HCl} \\
\mathrm{cem}\end{array}$} & \multirow{3}{*}{$\begin{array}{c}38 \% \text { ige } \\
\mathrm{HCl} \\
\mathrm{ccm}\end{array}$} & \multirow{3}{*}{$\begin{array}{c}10 \% / 0 \mathrm{jge} \\
\mathrm{BaCl}_{2} \\
\mathrm{ccm}\end{array}$} & \multicolumn{2}{|c|}{$\mathrm{Ba} \mathrm{SO}_{4}$} & \multirow{3}{*}{$\begin{array}{c}\text { Fehler } \\
\% \\
\end{array}$} \\
\hline & & & & & & 0 & C & \\
\hline & & & & & & 9 & $g$ & \\
\hline 1 & 25 & 75 & 10 & - & 5 & 0,3694 & 0,3668 & $+0,71$ \\
\hline 2 & 25 & 75 & 10 & - & 15 & 0.3691 & 0,3668 & $+0,63$ \\
\hline 3 & 25 & 75 & - & 10 & 10 & 0,5500 & 0,5457 & +0.79 \\
\hline
\end{tabular}

Im Niederschlag 1 wurde nach dem Schmelzen mit Soda nephelometrisch der Chlorgehalt bestimmt. Mau fand $0,9 \mathrm{mg}$, welche Menge 2,6 $\mathrm{mg}$ okkludiertem $\mathrm{BaCl}_{2}$ entspricht. Das korrigierte Endresultat wird also $0,3694-0,0026=0,3668 \mathrm{~g}$, was mit der wahren Menge übereinstimmt.

Niederschlag 2 war sehr fein. Vielleicht ist diese feinere Verteilung durch den grösseren Überschuss an Baryumchlorid hervorgerufen, dieselbe Erscheinung zeigte sich nämlich auch, wenn Baryumsulfat mit einer Baryumchloridlösung geschüttelt wurde.

Nr. 3 entspricht der Erwartung, obgleich hier der positive Fehler, der durch mehr Salzsäure verursacht wird, schon teilweise kompensiert zu sein scheint durch die grössere Löslichkeit des Baryumsulfats in der stark sauren Lösung. Wie schon erwähnt wurde, erhöhen starke Säuren die Löslichkeit des Baryumsulfats bedentend.

Aus dem Vorhergehenden schliessen wir also, dass man, wenn anders nur Sulfat anwesend ist, am besten die Korrektur für jeden Fall bestimmt durch Aufschliessen mit Soda, Auslaugen und nephelometrische Chlorbestimmung [Richards und Parker $\left.\left.{ }^{1}\right)\right]$.

$\mathrm{Hulett}$ und Duschak (l. c.) geben eine Methode an für die gravimetrische Bestimmung des Chlors, wofür aber eine besondere Apparatur erforderlich ist.

b) Nitrate. Wenn $\mathrm{NO}_{3}$-Ionen in der Lösung anwesend sind, macht man unter' allen Umständen einen grossen positiven Febler [Allen and Johnston ${ }^{2}$ ), Karaoglanow $\left.{ }^{3}\right)$ ].

1) Ztschr. f. anorg. Chem. 8, 43 (1894).

2) Ibid. 40, 196 (1904).

3) A. a. $\mathrm{O}$. 
Ruppin ${ }^{1}$ fand aber, dass Salpetersäure keinen Einfluss auf das Ergebnis. hatte. Aus verschiedenen Gründen sind jedoch seine Schlüsse bedeutungslos.

Auch hier weisen die Ergebnisse auf einen Chemismus hin, der analog ist mit der Okklusion von Baryumchlorid. Hier reagieren die $\mathrm{Ba} \mathrm{NO}_{3}$-Ionen mit $\mathrm{SO}_{4} "$

$$
2 \mathrm{Ba} \mathrm{NO}_{3}{ }^{\circ}+\mathrm{SO}_{4}{ }^{\prime \prime} \rightarrow \mathrm{Ba}_{2}\left(\mathrm{NO}_{3}\right)_{2} \mathrm{SO}_{4} \text {. }
$$

Dieser Komplex ist schwerer als $\mathrm{Ba}_{2} \mathrm{Cl}_{2} \mathrm{SO}_{4}$, so dass der Nitratfehler grösser ist. Der $\mathrm{NO}_{3}$-Fehler ist jedoch noch grösser als man aus dem Verhältnis schliessen sollte.

$$
\mathrm{NO}_{3} \text {-Fehler }=\frac{\mathrm{Ba}_{2}\left(\mathrm{NO}_{3}\right)_{2} \mathrm{SO}_{4}-\mathrm{Ba} \mathrm{SO}_{4}}{\mathrm{Ba}_{2} \mathrm{Cl}_{2} \mathrm{SO}_{4}-\mathrm{Ba} \mathrm{SO}_{4}} \times \mathrm{Ba} \mathrm{Cl}_{2} \text {-Fehler }
$$

Wahrscheinlich ist Baryumnitrat wenfger dissoziiert als Baryumchlorid. Wir haben versucht, auf folgende Weise den Fehler zu beseitigen.

Nach der Fällung gaben wir dem Niederschlag Gelegenheit, sich abzusetzen, wonach die Lösung durch ein Filter abgegọsen wurde. Hiernach gossen wir in das Becherglas $400 \mathrm{~cm}$ Wasser, erhitzten bis. zum Sieden, fügten Salzsäure und einige Kubikzentimeter Baryumchloridlösung hinzu und rührten dann mechanisch $2-6$ Stunden um. Wir hielten die Temperatur auf $90-95^{\circ}$, während das verdampfte Wasser èrsetzt wurde. Wir setzten voraus, dass bei Anwesenheit von einem kleinen Übermaßs an Baryụm-Ionen das $\mathrm{Ba}_{2}\left(\mathrm{NO}_{3}\right)_{2} \mathrm{SO}_{4}$ sich rasch in Baryumsulfat verwandeln würde. Wir fügten Salzsäure hinzu, weil offenbar bei gleichzeitiger Anwesenheit dieser Säure der Nitratfehler kleiner wurde.

Die Ergebnisse der Analyse sind in folgender Tabelle zusammengestellt:

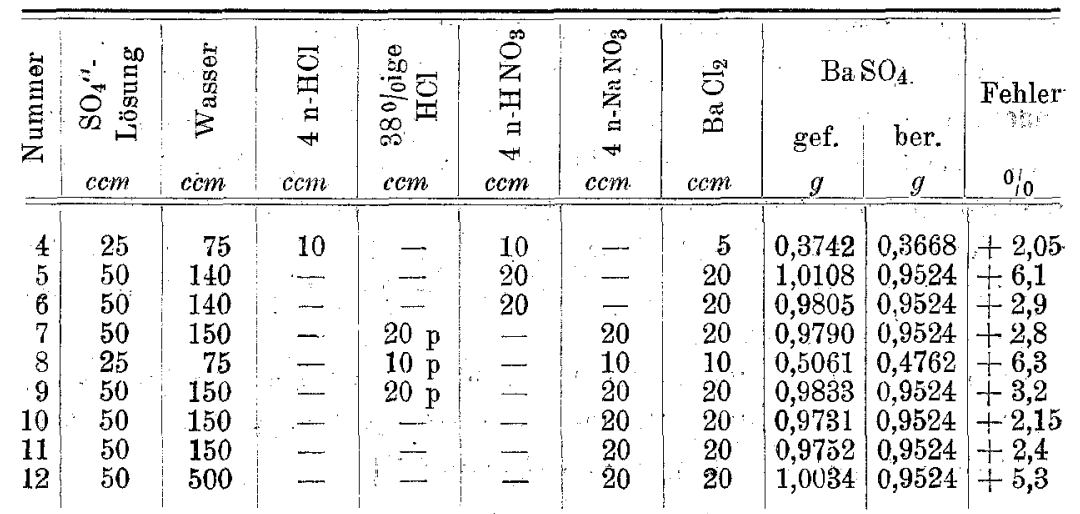

$\mathrm{p}$ bedeutet: hinzugefügt nach der Fâllung.

1) Diese Ztschr. 56, 417 (1917).

2) Chem. Ztg. 38, 17 (1909); diese Ztschr. 49, 406 (1900); 50, 180 (1901). 
Die Niederschläge 4 und 5 wurden nach der Fällung wie gewöhnlich behandelt, also nicht gerührt.

Nr. 6 haben wir nach der Fällung und nach dem Dekantieren 2 Stunden mit $300 \mathrm{ccm}$ Wasser, $10 \mathrm{~cm} 4 \mathrm{n}-\mathrm{HCl}$ and $3 \mathrm{ccm} 10 \%$ igem $\mathrm{Ba} \mathrm{Cl}_{2}$ wie angegeben gerührt.

Nr. 7 wurde nach der Fällung während 2 Stunden mit $20 \mathrm{~cm}$ $38 \%$ igem $\mathrm{HCl}$ gerührt und danach sofort filtriert, es ist also eine Wiederholung yon. Nr. 6 .

Nr. 8 ist nach der Fällung sofort filtriert.

Nr. $9=\mathrm{Nr} .7$, wurde aber 2 Stunden gerührt.

Nr. $10=$ Nr. 6, wurde aber 5 Stunden statt 2 gerührt.

Nr. 11 warde dekantiert, danach 3 Stunden lang mit 3 ccm $\mathrm{Ba} \mathrm{Cl}_{2}$ gerührt, abgegossen, wieder 3 Stunden mit $20 \mathrm{ccm} 38 \%$ iger $\mathrm{H} \mathrm{Cl}$ und Wasser gerührt and danach filtriert.

Nr. 12 wurde mit verdünntem Reagens in verdünnter $\mathrm{SO}_{4}$-Lösung gefällt, danach dekantiert und während 3 Stunden mit $20 \mathrm{ccm} 38 \%$ iger $\mathrm{HCl}, 5 \mathrm{ccm} \mathrm{Ba} \mathrm{Cl} 2$ und $175 \mathrm{ccm}$ Wasser gerührt.

Aus diesen Analysen geht deutlich hervor, dass das Rühren mit einer verdünnten $\mathrm{Ba} \mathrm{Cl}_{2}$-Lösung einen Einfluss auf die Ergebnisse ausübt und dass der Fehler kleiner wird, je länger man rührt (Nr. 10, 11, 7, 6). Dieses Ergebnis stützt unsere oben genannte Annahme.

Der Niederschlag Nr. 4 wurde mit Soda geschmolzen, mit angesäuertem Wasser aufgenommen und mit Zinkpulver reduziert.

Im Filtrat bestimmten wir das Ammonium kolorimetrisch. Wir fanden $0,5 \mathrm{mg} \mathrm{NH}_{4}$, was $3,7 \mathrm{mg}$ okkludiertem Baryumnitrat entspricht. Hierdurch war eine Abweichung von $1 \%$ erklärt. Der übrige Fehler muss also der $\mathrm{Ba} \mathrm{Cl}_{2}$-Okklusion zugeschrieben werden.

Aus der Tabelle ergibt sich, dass man, wenn man lange rübrt, den Fehler wohl verkleinern kann, aber man bekommt auf diese Weise kein richtiges Ergebnis. Man ist also gezwungen, entweder die Salpetersäure vor der Analyse durch Eindampfen auszutreiben oder im Niederschlag $\mathrm{Cl}^{\prime}$ und $\mathrm{NH}_{4}$ nach obigen Angaben zu bestimmen und die hieraus berechnete Korrektur vom gefundenen Gewicht abzuziehen. Das Ergebnis ist dann jedoch nicht mehr ganz zuverlässig. Im salzsauren Mittel erhielt man die günstigsten Resultate.

Gooch und Hill ${ }^{1}$ ) entfernten alle okkludierten Stofie, wie folgt: Der geglühte Niederschlag wird in warmer konzentrierter Schwefelsäure

1) Chem. Zentrbl. 1913, I, S. 136:; diese Ztschr. ä6, 417 (1917). 
gelöst, worin sich $10 \%$ des Baryumsulfats auflösen. Sie erhitzen die Lösung einige Zeit, dampfen zur Trockne und glühen, worauf alle okkludierten Stoffe ausgewaschen werden können. Okkludiertes Baryum wird jedoch so nicht entfernt.

c) Phosphate. Weil Baryumphosphat in einem nentralen Mittel unlöslich ist, können wir hier grosse positive Fehler erwarten.

Der Niederschlag besteht in diesem Fall aus einem Konglomerat von Baryumsulfat and Baryumphosphat. In saurer Lösung zeigt sich, dass der Niederschlag auch dann nicht phosphatfrei ist.

Cnter allen Umständen wurde denn auch immer in den Niederschlägen Phosphat nachgewiesen.

Wie der Erwartung entspricht, zeigte es sich, dass der Fehler bei steigender $\mathrm{PO}_{4}{ }^{\prime \prime}$-Konzentration zunimmt, was denn auch aus folgenden Analysen hervorgeht.

In untenstehender Tabelle bedeutet a Hinzufügung vor, p nach der Fällung.

Für die $\mathrm{PO}_{4}{ }^{\prime \prime}$-Lösung warde eine $6,6 \%$ ige Lösung von Ammoniumphosphat angewandt.

\begin{tabular}{|c|c|c|c|c|c|c|c|c|c|}
\hline \multirow[t]{2}{*}{$\mathrm{Nr}}$. & \multirow{2}{*}{$\begin{array}{c}\mathrm{SO}_{4}{ }^{\prime \prime}- \\
\text { Lösung } \\
\mathrm{ccm}\end{array}$} & \multirow{2}{*}{$\begin{array}{l}\mathrm{H}_{2} \mathrm{O} \\
\mathrm{ccm}\end{array}$} & \multirow{2}{*}{$\begin{array}{c}4 \mathrm{n}-\mathrm{HCl} \\
\mathrm{ccm}\end{array}$} & \multirow{2}{*}{$\begin{array}{c}38 \% \text { ige } \\
\mathrm{HCl} \\
\mathrm{ccm} \\
\end{array}$} & \multirow{2}{*}{$\begin{array}{c}\mathrm{PO}_{4}{ }^{\prime \prime \prime}- \\
\text { Lösung } \\
\text { ccm }\end{array}$} & \multirow{2}{*}{$\begin{array}{c}\mathrm{Ba} \mathrm{Cl}_{2} \\
\mathrm{~cm}\end{array}$} & \multicolumn{2}{|c|}{$\mathrm{Ba} \mathrm{SO}_{4}$} & \multirow{2}{*}{$\begin{array}{c}\text { Fehler } \\
\%\end{array}$} \\
\hline & & & & & & & 0 & $\mathrm{C}$ & \\
\hline 13 & 25 & 75 & 5 & - & 1 & 10 & 0,4875 & 0,4762 & $+2,4$ \\
\hline 14 & 25 & 75 & 5 & - & 5 & 10 & 0,4938 & 0,4762 & $+3,7$ \\
\hline 15 & 25 & 75 & 5 & $\ldots$ & 10 & 10 & 0,4946 & 0,4762 & $\begin{array}{r}+3,9 \\
\end{array}$ \\
\hline 16 & 25 & 75 & 5 & $10 \mathrm{a}$ & 5 & 10 & 0,5525 & 0,5425 & $+1,8$ \\
\hline 17 & 25 & 75 & 5 & $10 \mathrm{p}$ & 5 & 10 & 0,5550 & 0,5425 & $+2,3$ \\
\hline
\end{tabular}

Bei den Analysen 13, 14, 15 zeigt sich die Zunahme des Fehlers bei steigender $\mathrm{PO}_{4}{ }^{\prime \prime}$-Konzentration. Ausserdem zeigt sich bei 16 und 17, dass es besser ist, mehr Säure vor als nach der Fällung hinzuzufügen.

Wie Winkler ${ }^{1}$ ) angibt, und wie sich auch aus unseren Analysen ergibt, wird der Fehler klein, wenn man in ziemlich stark saurer Lösung fällt. W i l k l e r empfiehlt, in $10 \%$ iger Salzsäurelösung niederzuschlagen. Dann ist aber die Löslichkeit des Baryumsulfats bedeutend grösser, so dass diese Methode notwendigerweise auf der Kompensation von Fehlern beruhen muss und deshalb nicht empfehlenswert ist.

1) A. a. O. 
Wir haben daher versucht, das störende Ion vor der Fällung, wie folgt, aus der Lösung zu entfernen.

Dic Lösung wird auf dem Wasserbad erhitzt und mit Ammoniak übersättigt, wonach ein Überschuss an Magnesiumchlorid hinzugefügt wurde, wodurch die Phosphorsäure ausgefällt wurde. Hiernach gaben wir die $\mathrm{BaCl}_{2}$-Lösung tropfenweise hinzu, schliesslich säuerten wir die Lösung mit so viel $\mathrm{HCl}$ an, bis die Reaktion auf Methylorange sauer war, und fügten dann noch $1.0-20 \mathrm{~cm} 4 \mathrm{n}-\mathrm{HCl}$ hinzu, digerierten and filtrierten wie sonst.

Es zeigte sich, dass wir den Niederschlag auf diese Weise nicht phosphatfrei bekommen konnten.

Wir fanden folgende Zahlen:

\begin{tabular}{|c|c|c|c|c|c|c|}
\hline \multirow[b]{2}{*}{$\mathrm{Nr}$. } & \multirow{2}{*}{$\begin{array}{c}\mathrm{SO}_{4}{ }^{\prime \prime}- \\
\text { Lösung } \\
\text { ccm }\end{array}$} & \multirow[b]{2}{*}{$\begin{array}{c}\text { Wasser } \\
\text { ccm }\end{array}$} & \multirow{2}{*}{$\begin{array}{c}6,6 \% \text { iges } \\
\text { Ammon.- } \\
\text { Phosphat } \\
\mathrm{ccm}\end{array}$} & \multicolumn{2}{|c|}{$\mathrm{BaSO}_{4}$} & \multirow{2}{*}{$\begin{array}{c}\text { Fehler } \\
0 / 0\end{array}$} \\
\hline & & & & $\begin{array}{c}\text { gefunden } \\
g\end{array}$ & $\begin{array}{c}\text { berechnet } \\
g\end{array}$ & \\
\hline 18 & 25 & 70 & 5 & 0,5374 & 0,5272 & $+1,9$ \\
\hline 19 & 25 & 70 & 10 & 0,5385 & 0,5272 & $+2,1$ \\
\hline 20 & 25 & 70 & 15 & 0,5352 & 0,5272 & $+1,3$ \\
\hline 21 & 25 & 70 & 25 & 0,5452 & 0,5272 & $+3,5$ \\
\hline
\end{tabular}

Der Erwartung gemäls wạr der Fehler in 19 wieder grösser als der von 18. Nr. 21 ist auch nach der Regel.

20 wurde noch einmal mit $25 \mathrm{~cm} 4 \mathrm{n}-\mathrm{H} \mathrm{Cl}$ ausgewaschen, wodurch der geringere Fehler durch Kompensation erklärt wird.

Hiernach wurden noch folgende Versuche angestellt.

Die Sulfat- und Phosphatlösung (neutral) wurde mit einem Überschuss an Aluminiumchloridlösung gekocht, darauf wurde Ammoniak hinzugefügt, bis die Reaktion auf Methylrot alkalisch war. In dieser alkalischen Lösung wurde $\mathrm{SO}_{4}$ mit $10 \%$ iger Baryumazetatlösung gefällt, wonach man die Lösung wie oben behandelte (man säuerte an usw.). Ausserdem stellte man auf diese Weise noch einen Versuch an mit Chlorzinklösung, wobei die Flüssigkeit erst ammoniakaliseh gemacht wurde.

\begin{tabular}{c|c|c|c|c|c|c}
\hline \hline Nr. & $\begin{array}{c}\mathrm{SO}_{4}{ }^{\prime \prime}- \\
\text { Lösung }\end{array}$ & Wasser & $\begin{array}{c}\text { Phosphor- } \\
\text { säure }\end{array}$ & \multicolumn{2}{|c|}{ Ba SO } \\
gefunden & berechnet & Fehler \\
& $c c m$ & $c c m$ & $c c m$ & $g$ & $g$ & 0 \\
\hline \hline 22 & 25 & 70 & 10 & 0,5430 & 0,5272 & $+3,0$ \\
23 & 25 & 70 & 15 & 0,5420 & 0,5272 & $+2,8$ \\
24 & 25 & 70 & 20 & 0,5580 & 0,5272 & $+5,8$
\end{tabular}


Aus diesen Versuchen geht hervor, dass in einer phosphorsäurehaltigen, Lösung keine genaue $\mathrm{SO}_{4}$-Bestimmung ausgeführt werden kann.

Die bestehenden Metloden (u. a. Winkler), beruhen auf der Kompensation von Fehlern. Es ist also empfehlenswert, bei genauen Bestimmungen das Phosphat zu entfernen.

d) Kalzium. van 't Kruys ${ }^{1}$ ) und Winkler ${ }^{3}$ ) fanden, dass Ca-Ionen in neutraler Lösung das Ergebnis vermindern. Ersterer empfielilt, nach der Fällnng viel starke Salzsäure hinzuzufügen, dann zu kochen und den Überschuss an Särre wieder mit Ammoniak zu neutralisieren.

Winkler entfernt das Ca-Ion mit Soda oder er arbeitet in einem Mittel, das $10 \% \mathrm{H} \mathrm{Cl}$ enthält.

Die Methode van 't Kruys kann ebenso wie die von Winkler auf der Löslichkeit des Niederschlags in der starken Säure beruhen. Hierdurch wird der Fehler durch Okklusion von Kalzium- oder Baryumchlorid kompensiert. Bei der Neutralisation (van 't Kruys) wird nämlich nicht alles gelöste Baryumsulfat ausfallen, weil es sehr leicht in übersättigter Lösung bleibt. Ausserdem ist die Löslichkeit desselben in konzentriertem Ammoniumchlorid ziemlich gross.

In schwach saurem Mittel fanden wir einen negativen Fehler, der mit zunebmender Ca-Ionenkonzentration grösser wurde. Hier haben wir es augenscheinlich mit einer teilweisen Fällung von Kalziumsulfat statt Baryumsulfat zu tun. Wenn die Lösung jedoch stark sauer war, fanden wir einen ziemlich grossen positiven Fehler, der wahrscheinlich durch Okklusion von Kalzium- und Baryumchlorid entstand. Wenn wir nach van 't Kruys arbeiteten, war der Fehler viel geringer, bis $+0,25 \%$.

Schliesslich fanden wir, dass die Ergebnisse verhältnismäfsig gut ausfallen, wenn man nach Ansäuern mit Essigsäure bei Siedehitze mit Baryumazetat als Reagens fällt. Diese Methode liefert richtige Ergebnisse bei Anwesenheit von nicht zu grossen Mengen Kalzium.

In folgender Tabelle bedeutet a: Säure vor der Fällung, $p$ : danach hinzugefügt; n: neutralisiert. Es wurden immer $10 \mathrm{ccm}$ Reagens hinzugefügt.

1) Diese Ztschrft. 49, 393 (1910).

2) A. a. 0 . 


\begin{tabular}{|c|c|c|c|c|c|c|c|c|c|c|}
\hline 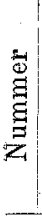 & 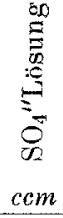 & $\begin{array}{l}0 \\
\text { I } \\
I\end{array}$ & $\begin{array}{c}\bar{O} \\
\mathbb{I} \\
\overline{7} \\
\cdot+ \\
\mathrm{ccm}\end{array}$ & $\begin{array}{l}\overrightarrow{0} \\
\mathbb{Z} \\
0 \\
0 \\
0 \\
0 \\
0 \\
\infty \\
6 \\
\mathrm{ccm}\end{array}$ & 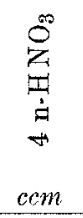 & 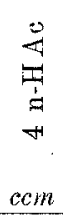 & 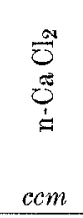 & $\begin{array}{c}\text { gefunden } \\
g \\
\end{array}$ & $\begin{array}{c}\text { berechnet } \\
g\end{array}$ & Fehler \\
\hline 25 & 25 & 70 & $10 \mathbf{a}$ & - & - & - & 1 & 0,3657 & 0,3668 & $-0,3$ \\
\hline 26 & 25 & 70 & $10 \mathrm{a}$ & - & - & - & 5 & 0,3642 & 0,3668 & $-0,7$ \\
\hline 27 & 25 & 70 & $10 \mathrm{a}$ & - & - & - & 10 & 0,3626 & 0,3668 & $-1,15$ \\
\hline 28 & 25 & 70 & - & $10 a$ & - & - & 10 & $0,55: 24$ & 0,5457 & $+1,3$ \\
\hline 29 & 25 & 70 & - & $10 \mathrm{a}$ & - & - & 10 & 0,5546 & 457 & $+1,5$ \\
\hline 30 & 25 & 70 & - & $10 a$ & - & - & 10 & 0,5535 & 0,5457 & $+1,3$ \\
\hline 31 & 25 & 70 & - & $10 p$ & - & - & 10 & 0,5484 & 0,5457 & $+0,4$ \\
\hline 32 & 25 & 70 & - & $10 \mathrm{pn}$ & - & - & 10 & 0,5476 & 0,5457 & $+0,25$ \\
\hline 33 & 25 & 70 & - & - & - & - & 10 & 0,5504 & 0,5457 & $+0,85$ \\
\hline 34 & 25 & 70 & $10 p$ & - & - & - & 10 & 0,5506 & 0,5457 & $+0,9$ \\
\hline 35 & 25 & 70 & - & - & $10 \mathrm{a}$ & - & 10 & 0,5781 & 0,5457 & $+5,8$ \\
\hline 36 & 25 & 70 & $\cdots$ & - & $5 \mathrm{a}$ & - & 10 & 0,5801 & 0,5457 & $+6,2$ \\
\hline 37 & 25 & 70 & - & - & $2 a$ & - & 10 & 0,5571 & 0,5457 & $+2,0$ \\
\hline 38 & 25 & 75 & $10 a$ & - & - & - & 10 & 0,5562 & 0,5457 & $+1,8$ \\
\hline 39 & 25 & 75 & - & - & - & - & 10 & 0,5635 & 0,5457 & $+3,1$ \\
\hline 40 & 25 & 75 & - & $10 \mathrm{a}$ & - & - & 10 & 0,4933 & 0,4762 & $+3,6$ \\
\hline 41 & 25 & 75 & - & $10 \mathrm{p}$ & - & - & 10 & 0,4842 & 0,4762 & $+1,7$ \\
\hline 42 & 25 & 70 & - & - & - & - & 10 & 0,4942 & 0,4762 & $+3,8$ \\
\hline 43 & 25 & 70 & - & 一 & - & 5 & 10 & 0,4781 & 0,4762 & +0.4 \\
\hline 44 & 25 & 70 & - & - & - & 15 & 10 & 0,4778 & 0,4762 & $+0,3$ \\
\hline 45 & 25 & 70 & 5 & - & - & - & $\begin{array}{c}\mathrm{n}-\mathrm{C} \mathrm{c} \mathrm{Ac} \\
5\end{array}$ & 0,5245 & 0,5272 & $-0,5$ \\
\hline 46 & 25 & 70 & 5 & - & - & $\ldots$ & 10 & 0,5243 & 0,5272 & $-0,5$ \\
\hline 47 & 25 & 70 & 5 & - & - & $\longrightarrow$ & 15 & 0,5240 & 0,5272 & $-0,6$ \\
\hline 48 & 25 & 70 & -5 & - & - & - & 25 & 0,5157 & 0.5272 & $-2,2$ \\
\hline
\end{tabular}

Nr. 38, 39, 40, 41 waren kalt gefällt, danach gekocht.

Bei 42-48 wurde als Reagens $10 \%$ iges Baryumazetat benutzt.

Man handelt unseres Erachtens am besten, wenn man in essigsaurer Jösung mit Baryumazetat fällt.

Es zeigte sich, dass die geglühten Niedersehläge 43 und 44 stark alkalisch auf Phenolphtalein reagierten. Dies wird durch die Okklusion von Kalziumazetat oder Baryumazetat erklärt, wobei dann beim Erbitzen das Azetat zerlegt wird und die Oxyde zurückbleiben. Zusammenfassend finden wir also, dass die Gegenwart von Ca-Ionen zu zwei Fehlern Anlass geben kann, die von dem Säuregrad der Lösung abhängig sind, nämlich : 
In schwach salzsaurer Lösung ein negativer Febler, der dadurcls entsteht, dass Kalziumsulfat mit ausfällt, welcher Fehler der Kalziumkonzentration entsprechend zunimmt.

In stark salz- und essigsaurer Lösung ein positiver Fehler durch Okklusion der Elektrolyten.

Beide Fehler sind also die Folgen eines chemischen Vorgangs.

e) Ferriverbindungen. Nach Küster und Thiel ${ }^{1}$ ) ist die Störung durch Ferrisalze der Bildung des komplexen $\mathrm{Fe}\left(\mathrm{SO}_{4}\right)_{2}{ }^{\prime \prime}$-Ions. zuzuschreiben, was derselben Erscheinung entspricht, die Recoura ${ }^{2}$ ) für Chromverbindungen wahrnahm.

Die Resultate werden hierdurch zu niedrig, weil $\mathrm{Ba}\left[\mathrm{Fe}\left(\mathrm{SO}_{4}\right)_{2}\right]_{2}$ entsteht, das mit $4 \mathrm{BaSO}_{4}$ äquivalent ist. Ein bedeutender negativer Feller ist also erklärlich.

$\mathrm{K}$ üster und Thiel fanden, dass die Reaktion zwischen $\mathrm{Ba}$ ", $\mathrm{Fe}^{\prime \prime}$ und $\mathrm{SO}_{4}$ " eine Zeitreaktion ist. Dies wird aus der langsamen Hydrolyse von Ferrisalzen erklärt.

Bei Fällung in der Kälte war der Fehler nahezu gleich Null, dies. lässt sich aber wieder aus der Kompensation von Fehlern erklären. Überdies fanden sie, dass Hinzufügen von Salzsäure den Fehler vermindert; auch diese Erscheinung erklärt sich aus der Zunahme der Okklusion von Baryumchlorid.

Heidenreich ${ }^{3}$ ) und Meineke $\mathrm{e}^{4}$ ) behaupten, dass nach Reduktion: zu Ferro-Ion der Fehler beseitigt ist. Küster und Thiel ${ }^{5}$ ) stimmten ihnen nicht bej, aber, wie sie selbst schon bemerkten, machten sie den Fehler, zu viel Zink für die Reduktion zu benutzen, wodurch ein neuer Fehler eintritt [s. Lunge und Stierlin ${ }^{6}$ )].

Winkle ${ }^{7}$ ) empfieblt denn auch, für die Reduktion Hydroxylamin anzuwenden.

1) Ztschr. f. anorg. Chem. 19, 97 (1899) und 21, 73, 194 (1899); dieseZtschrft. 38, 209, 352 (1899).

2) Compt. rend. 113, 857 und 114, 477; vergl. diese Ztschrft. 38, 243 (1899).

3) Ztschrft. f. anorg. Chem. 20, 283 (1899); vergl. diese Ztschrft. 38, $351(1899)$.

4) Diese Ztschrft. 38, 351 (1 $\$ 99)$.

5) Ztschrft. f. anorg. Chem. 36, 84 (1903).

6) Ztschrft.'f. angew. Chem. 18, 21 (1906); diese Ztsehrft. 45, 714 (1906).

7) A. a. O. 
K üster und Thiel kamen so zu ihrer Methode, das Eisen mit Ammoniak zu entfernen und danach das $\mathrm{SO}_{4}{ }^{\prime \prime} \mathrm{zu}$ făllen [siehe auch Richards $\left.{ }^{1}\right)$ ].

Jannasch and Richards ${ }^{2}$ ) entfernten das Ferri-Ion; indem sie nach Umschmelzen des Niederschlags mit Soda und Auslaugen im Filtrat das $\mathrm{SO}_{4}$ " bestimmten.

Karaoglanow ${ }^{3}$ ) erhielt richtige Ergebnisse bei Anwesenheit von Ferri-Ion indem er aus stark verdünnten sauren Lösungen niederschlug.

In untenstehender Tabelle sind unsere Ergebnisse zusammengefasst. Die letzten drei Analysen sind nach $K$ ü s ter und Thi el, wie folgt, ausgeführt.

Die neutrale Iıösung wurde bis zum Sieden erhitzt, wonach das Eisen mit $10 \%$ igem Ammoniak gefällt wurde. Ein Teil des kolloidalen Eisenoxydhydrats flockte während der Erhitzung unter Einfluss des Sulfations schon aus.

Unter vorsichtigem Erhitzen auf einem Wasserbad wurde das Reagens hinzutropfen gelassen. Hierauf säuerte man mit Salzsäure auf Methylorange an (die Lösung verliert dann die braune Farbe uud wird hellgelb), wonach man $10-20 \mathrm{ccm} 4 \mathrm{n}-\mathrm{H} \mathrm{Cl}$ hinzufügte und die $\mathrm{Be}$ handlung weiter wie gewöhnlich fortsetzte.

In folgender Tabelle bedeutet $\mathrm{EA}=$ Äthylalkohol und $\mathrm{BA}=$ Isobutylalkohol.

\begin{tabular}{|c|c|c|c|c|c|c|c|c|c|c|}
\hline $\mathrm{Nr}^{2}$. & $\begin{array}{c}\mathrm{SO}_{4}{ }^{\prime \prime}- \\
\mathrm{Lösung} \\
\text { ccm }\end{array}$ & $\begin{array}{l}\mathrm{H}_{2} \mathrm{O} \\
\mathrm{ccm}\end{array}$ & $\begin{array}{c}4 \mathrm{n}-\mathrm{HCl} \\
\mathrm{ccm}\end{array}$ & $\begin{array}{c}38 \% \text { ige } \\
\mathrm{HCl} \\
\mathrm{ccm}\end{array}$ & $\begin{array}{l}\mathrm{EA} \\
\mathrm{ccm}\end{array}$ & $\begin{array}{l}\mathrm{BA} \\
\mathrm{ccm}\end{array}$ & $\begin{array}{c}\mathrm{n}-\mathrm{Fe} \mathrm{Cl}_{3} \\
\mathrm{ccm}\end{array}$ & $\begin{array}{c}\text { Ba } \\
\text { gef. } \\
g \\
\end{array}$ & $\begin{array}{c}\mathrm{SO}_{4} \\
\text { ber: } \\
g\end{array}$ & $\begin{array}{c}\text { Fehler } \\
\%\end{array}$ \\
\hline 49 & 25 & 75 & 10 & - & $\stackrel{-}{ }$ & - & 1 & 0,3568 & 0,3668 & $-2,7$ \\
\hline 50 & 25 & 75 & 10 & - & - & - & 5 & 0,3560 & 0,3668 & $-2,9$ \\
\hline b̆1 & 25 & 75 & 10 & - & - & - & 10 & 0,3570 & 0,3668 & $-2,7$ \\
\hline 52 & 25 & 75 & 10 & - & 20 & - & 10 & 0,3638 & 0,3668 & $-0,8$ \\
\hline 53 & 25 & 75 & 10 & - & - & 5 & 5 & 0,3639 & 0,3668 & $-0,8$ \\
\hline 54 & 25 & 75 & - & 10 & - & - & 5 & 0,3405 & 0,3668 & $-7,1$ \\
\hline 55 & 25 & 75 & - & 10 & - & 5 & 5 & 0,3463 & 0,3668 & $-5,6$ \\
\hline 56 & 25 & 75 & 5 & - & - & - & 5 & 0,5502 & 0,5457 & $+0,8$ \\
\hline 57 & 25 & 75 & 5 & - & - & 5 & 5 & 0,5524 & 0,5457 & $+1,2$ \\
\hline 58 & 25 & 60 & - & - & - & $\ldots$ & 5 & 0,5455 & 0,5432 & $1+0,42$ \\
\hline 59 & 25 & 60 & - & - & - & - & 10 & $0,5 \pm 50$ & 0,5432 & $1+0,31$ \\
\hline 60 & 25 & 60 & - & - & - & - & 25 & 0,5453 & 0,5432 & $1+0,39$ \\
\hline
\end{tabular}

1) Ztschrft. f. anorg. Chem. 23, 383 (1900).

2) Journ. f. prakt. Chem. 39, 321 (1899); diese Ztschrft. 38, 209 (1899).

3) Diese Ztschrft. 56, 417 (1917). 
Aus diesen Ergebnissen folgt, dass die Fehler sowohl positiv als negativ sein können. Wenn man aus sehr schwach sauren Lösungen fällt, herrscht die Baryumchlorid-Okklusion vor, auch fällt dann das kolloidale Eisenoxyd mit, weil in verdünnten Säuren und bei hoher Temperatur die Ferrisalze ziemlich stark hydrolytisch gespalten sind, die Ergebnisse werden also zu hoch (Nr. 56 und 57). Da Alkohol die Dissoziation von Baryumchlorid vermindert, ist der Fehler bei 57 grösser als bei 56. In stärker saurer Lösung wird der Fehler negativ durch die Bildung des komplexen Sulfats. Hier üben Äthyl- und Isobutylalkohol gerade einen günstigen Einfluss aus, was wahrscheinlich der Kompensation durch Okklusion von mehr Baryumchlorid zuznschreiben ist.

Wenn man das Eisen erst mit Ammoniak fällt, werden nahezu richtige Ergebnisse gefunden $(58,59,60)$. Der positive Fehler bei letzteren Analysen wird ebenfalls durch Okklusion von Baryumchlorid erklärt, wir konnten nämlich in den Niederschlägen Chlor nachweisen.

Alle Niederschläge, mit Ausnahme der drei letzten, waren mehr oder weniger farbig, hellgelb bis orange, bisweilen grau durch Bildung von Schwefeleisen. Je grösser der negative Fehler war, desto stärker war der Niederschlag gefärbt.

f) Andere Metalle. Aluminium stört wenig. Creighton ${ }^{1}$ ) fand positive Fehler bei Anwesenheit dieser Metalle. Winkler fand jedoch negative, der von ihm gefundene Fehler ist aber sehr gering.

Was die Analyse von Kalialaun $\left[\mathrm{K}_{2} \mathrm{SO}_{4}, \mathrm{Al}_{2}\left(\mathrm{SO}_{4}\right)_{3} .24 \mathrm{aq}\right]$ betrifft, so konnten wir seiner Wahrnehmung beistimmeñ; hierbei fanden wir die Fehler -- 0,4 and $-0,5 \%$. Es ist jedoch nicht unwahrscheinlich, dass bei der Analyse von Alaun der negative Fehler des Kaliums durch den positiven des Aluminiums nach Creighton teilweise kompensiert wird, so dass ein kleiner negativer Fehler übrig bleibt.

Wir fanden in Übereinstimmung mit andern Autoren (van 't $\mathrm{Kr}$ u ys, Ruppin 1. c.), dass Kalium das Resultat erniedrigt, wie u. a. aus folgender Analyse hervorgeht:

\begin{tabular}{|c|c|c|c|c|c|c|c|}
\hline Nr. & $\begin{array}{c}\mathrm{SO}_{4}{ }^{\prime \prime} \text {-Lösung } \\
\mathrm{ccm}\end{array}$ & $\begin{array}{c}\text { Wasser } \\
. \\
\mathrm{ccm}\end{array}$ & $\begin{array}{c}4 \mathrm{n}-\mathrm{HCl} \\
\mathrm{ccm}\end{array}$ & $\begin{array}{c}4 \mathrm{n}-\mathrm{KOl} \\
\mathrm{ccm}\end{array}$ & $\begin{array}{c}\mathrm{Ba} \\
\text { gef. } \\
g\end{array}$ & $\begin{array}{c}\mathrm{SO}_{4} \\
\text { ber. }\end{array}$ & $\begin{array}{c}\text { Fehler } \\
0 \%\end{array}$ \\
\hline 61 & 25 & 75 & 10 & 10 & 0,3618 & 0,3668 & $-1,3$ \\
\hline
\end{tabular}

1) Ztschrft. f. anorg. Chem. 63, 58 (1909). 
Allen und $J$ ohnston fanden diese erniedrigende Wirkung nicht.

Der Fehler wird durch folgende Reaktion erklärt:

$$
2 \mathrm{KSO}_{4}^{\prime}+\mathrm{Ba}^{\prime \prime} \rightarrow \mathrm{BaK}_{2}\left(\mathrm{SO}_{4}\right)_{2} .
$$

Wenn wenig Natriumchlorid anwesend war, hatte dies kaum Einfluss auf das Resultat; grosse Mengen Natriumchlorid setzen es ebenfalls herab.

Die Wirkung anderer Metalle baben wir nicht mehr geprüft. Hierfür verweisen wir auf die oben erwähnten Arbeiten von Allen and Johnston, van 't Kruys, Winkler and Karaoglanow. Zusammenfassung der Ergebnisse:

1. Die Löslichkeit von Baryumsulfat, die bei gewöhnlicher Temperatur in Wasser $2,3 \mathrm{mg}$ pro Liter beträgt, nimmt mit der Temperatur stark zu.

Durch Salzsäure und Salpetersäure wird die Löslichkeit bedeutend erhöht, und zwar durch letztere mehr als durch erstere. Dies stimmt mit unseren Analysen, dass Salpetersäure mehr stört als Salzsäure, überein.

2. Bei Veraschen des trockenen Niederschlags im Porzeilantiegel findet fast keine Reduktion statt, dagegen eine sehr starke im Platintiegel. Es ist jedenfalls empfehlenswert, den Niederschlag mit einigen Tropfen Schwefelsäure zu behandeln und danach wieder zu glühen.

3. Die Behauptung Pregls, dass man die okkludierten Stoffe nach dem Glühen auswaschen kann, ist unrichtig.

4. Die Okklasion von Baryumchlorid, -Nitrat, Kalzium-, Ferri- und Kalium-Ionen muss bauptsächlich eine chemische Ursache haben.

5. Die Störung, welche ein Phosphat verursacht, entsteht durch. die Bildung von Baryumphosphat neben Baryumsulfat.

6. Es ist unmöglich allgemeine Vorschriften für die Sulfatbestimmung als Baryumsulfat in beliebigen Lösungen aufzustellen.

Utrecht.

Pharmaz. Lab. der Univ. 